\title{
Attention Modulates TMS-Locked Alpha Oscillations in the Visual Cortex
}

\author{
ㄱim D. Herring, ${ }^{1}$ Gregor Thut, ${ }^{5}$ @Ole Jensen, ${ }^{1}$ and $\odot$ Til 0. Bergmann ${ }^{1,2,3,4}$ \\ ${ }^{1}$ Donders Institute for Brain, Cognition and Behaviour, Radboud University Nijmegen, 6525 EN Nijmegen, The Netherlands, ${ }^{2}$ Institute of Psychology, \\ Christian-Albrechts University of Kiel, 24118 Kiel, Germany, ${ }^{3}$ Department of Neurology and Stroke, and Hertie Institute for Clinical Brain Research, and \\ ${ }^{4}$ Institute for Medical Psychology and Behavioral Neurobiology, University of Tübingen, 72076 Tübingen, Germany, and ${ }^{5}$ Centre for Cognitive \\ Neuroimaging, Institute of Neuroscience and Psychology, University of Glasgow, Glasgow G12 8QQ, United Kingdom
}

Cortical oscillations, such as $8-12 \mathrm{~Hz}$ alpha-band activity, are thought to subserve gating of information processing in the human brain. While most of the supporting evidence is correlational, causal evidence comes from attempts to externally drive ("entrain") these oscillations by transcranial magnetic stimulation (TMS). Indeed, the frequency profile of TMS-evoked potentials (TEPs) closely resembles that of oscillations spontaneously emerging in the same brain region. However, it is unclear whether TMS-locked and spontaneous oscillations are produced by the same neuronal mechanisms. If so, they should react in a similar manner to top-down modulation by endogenous attention. To test this prediction, we assessed the alpha-like EEG response to TMS of the visual cortex during periods of high and low visual attention while participants attended to either the visual or auditory modality in a cross-modal attention task. We observed a TMS-locked local oscillatory alpha response lasting several cycles after TMS (but not after sham stimulation). Importantly, TMS-locked alpha power was suppressed during deployment of visual relative to auditory attention, mirroring spontaneous alpha amplitudes. In addition, the early N40 TEP component, located at the stimulation site, was amplified by visual attention. The extent of attentional modulation for both TMS-locked alpha power and N40 amplitude did depend, with opposite sign, on the individual ability to modulate spontaneous alpha power at the stimulation site. We therefore argue that TMS-locked and spontaneous oscillations are of common neurophysiological origin, whereas the N40 TEP component may serve as an index of current cortical excitability at the time of stimulation.

Key words: alpha oscillations; auditory attention; TMS-EEG; TMS-evoked potential; transcranial magnetic stimulation; visual attention

Significance Statement

Rhythmic transcranial magnetic stimulation (TMS) is a promising tool to experimentally "entrain" cortical activity. If TMS-locked oscillatory responses actually recruit the same neuronal mechanisms as spontaneous cortical oscillations, they qualify as a valid tool to study the causal role of neuronal oscillations in cognition but also to enable new treatments targeting aberrant oscillatory activity in, for example, neurological conditions. Here, we provide first-time evidence that TMS-locked and spontaneous oscillations are indeed tightly related and are likely to rely on the same neuronal generators. In addition, we demonstrate that an early local component of the TMS-evoked potential (the N40) may serve as a new objective and noninvasive probe of visual cortex excitability, which so far was only accessible via subjective phosphene reports.

\section{Introduction}

Cortical oscillations reflect the synchronization of large neuronal populations, rhythmically shifting between states of excitability
(Schroeder and Lakatos, 2009). By modulating synaptic input and synchronizing neuronal output (Varela et al., 2001; Fries, 2005), they may temporally organize information processing and
Received May 1, 2015; revised Aug. 29, 2015; accepted Sept. 2, 2015.

Author contributions: J.D.H., G.T., 0.J., and T.O.B. designed research; J.D.H. performed research; J.D.H. analyzed data; J.D.H., G.T., O.J., and T.O.B. wrote the paper.

This work was supported by The Netherlands Organization for Scientific Research, VICI Grant 453-09-002, ALW Open Competition Grant 822-02-011, and Wellcome Trust Award Grant 098434 to G.T.

The authors declare no competing financial interests.

This article is freely available online through the J Neurosci Author Open Choice option.
Correspondence should be addressed to Dr. Til 0. Bergmann, Institute of Psychology, Christian-AlbrechtsUniversity of Kiel, Olshausenstrasse 62, 24118 Kiel, Germany. E-mail: bergmann@psychologie.uni-kiel.de. DOI:10.1523/JNEUROSCI.1833-15.2015 Copyright $\odot 2015$ Herring et al.

This is an Open Access article distributed under the terms of the Creative Commons Attribution License Creative Commons Attribution 4.0 International, which permits unrestricted use, distribution and reproduction in any medium provided that the original work is properly attributed. 
communication between brain areas and thus support a variety of cognitive functions (VanRullen and Koch, 2003; Buzsáki and Draguhn, 2004; Engel and Fries, 2010; Hanslmayr et al., 2011; Jensen et al., 2014). Yet, although numerous studies have linked cortical oscillations to task performance, the causal relevance of these oscillations remains to be proven (Thut et al., 2012).

To this end, neuronal oscillations need to be experimentally manipulated to investigate their immediate impact on behavior. Research in humans can benefit from noninvasive brain stimulation techniques, such as transcranial magnetic stimulation (TMS) and transcranial alternating current stimulation (tACS), to directly induce frequency-specific rhythmic activity in the brain (Thut et al., 2011a; Herrmann et al., 2013). Whereas entrainment effects of tACS supposedly rely on subtle shifts in neurons' membrane potential, rTMS is capable of periodically triggering action potentials, and supposedly drive neuronal oscillations (Thut et al., 2011a). Accordingly, short trains of rTMS in the $8-14 \mathrm{~Hz}$ alpha range were found to produce frequencyspecific effects on task performance (Klimesch et al., 2003; Sauseng et al., 2009; Romei et al., 2010; Romei et al., 2011; Jaegle and Ro, 2014; Ruzzoli and Soto-Faraco, 2014) in line with the supposed cognitive function of alpha oscillations as rhythmic inhibition of task-irrelevant brain regions (Klimesch et al., 2007; Jensen and Mazaheri, 2010). However, evidence for actual "entrainment" in electrophysiology is still sparse (Thut et al., 2011b; Hanslmayr et al., 2014). The question remains open whether oscillatory responses locked to transcranial stimulation and spontaneously occurring neuronal oscillations originate from the same neuronal generator.

Supporting the notion of a common mechanism for TMSlocked and spontaneous oscillations, the frequency profile of TMS-evoked potentials (TEPs) demonstrates site and state specificity resembling that of spontaneous oscillations: First, it predominates at the frequency of neuronal oscillations naturally generated in the stimulated brain region (e.g., in the alpha range for the visual cortex) (Rosanova et al., 2009; Garcia et al., 2011). Second, it depends on the current state of vigilance (e.g., wake vs deep sleep) (Massimini et al., 2007; Bergmann et al., 2012).

We argue that, if TMS-locked alpha oscillations in the visual cortex resemble spontaneous alpha oscillations, they should both be subject to top-down control (i.e., suppressed when visual attention is high but boosted when visual attention is low, as during endogenous cross-modal shifts of attention) (Adrian, 1944; Fu et al., 2001). In contrast, early TEP components, reflecting immediate cortical excitation, should depend on visual cortex excitability itself and therefore show the opposite pattern. To test these hypotheses, we applied single-pulse TMS to the left visual cortex (intermingled with Sham TMS to the left shoulder to control for auditory and somatosensory input) while participants performed a cross-modal detection task, requiring them to attend to a visual while ignoring an auditory input stream (High visual attention) or vice versa (Low visual attention).

\section{Materials and Methods}

Participants. Participants were recruited from a participant database from the Radboud University. Twenty-four healthy subjects with normal or corrected-to-normal vision ( 15 females, 9 males) participated in the experiment. All subjects conformed to standard inclusion criteria for MRI, EEG, and TMS. Written informed consent was gathered at the start of the experiment according to the Declaration of Helsinki. The study was approved by the local ethics committee. Participants were compensated financially at a rate of 10 euros per hour.

Procedure. All subjects participated in an intake session and one experimental session on 2 separate days. During the intake session, a structural
MRI scan was obtained for subsequent neuronavigation of TMS. Participants were familiarized with the cross-modal attention task followed by the determination of TMS intensity and location as well as titration of visual and auditory stimulus parameters using an adaptive staircase procedure (see below). During the experimental session, subjects were first prepared for EEG recordings and TMS neuronavigation. Then, resting state EEG was recorded for $2 \mathrm{~min}$ with eyes open and $2 \mathrm{~min}$ with eyes closed, before participants performed a 5 min training session of the cross-modal attention task while the EEG was recorded but without applying TMS. Afterward, the cross-modal attention task was performed in short blocks of 15-60 s alternating between conditions (see below) while EEG was recoded and TMS was applied (main experiment). Every 5 min, short breaks were introduced (Fig. 1A).

Attention task. Participants performed a blocked cross-modal attention task (Fig. 1A). Each block began with a brief audio-visual cue (i.e., the spoken and written words "Listen" or "Look") instructing the participants to pay attention to either the visual or the auditory background noise stream. They were presented simultaneously via a 17 inch TFT-monitor (resolution: $1024 \times 768$; refresh rate: $60 \mathrm{~Hz}$; viewing distance: $60 \mathrm{~cm}$ ) and earphones, respectively. In addition, the color of a central fixation dot indicated the block type throughout the entire experiment (green represents attend visual; red represents attend auditory) to prevent potential confusion. Participants had to detect subtle "targets" only in the attended background noise stream while ignoring changes in the other as distracting stimuli have been shown to increase alpha modulation (Haegens et al., 2012). Cue validity was thus always $100 \%$. For the visual background noise, the grayscale value of each pixel on the screen was randomly drawn from a uniform distribution. Visual targets consisted of small circular patches, 4 degrees visual angle in size, whose pixels were increased in contrast compared with the background (Fig. $1 B$ ) for a period of $500 \mathrm{~ms}$. The patches could appear at any location on the screen (except for a one patch-wide margin, 4 degrees visual angle, along the edge of the display and around the fixation dot; i.e., between 4 and 8 degrees vertically and 4 to 13 degrees horizontally relative to the fixation dot). Participants were informed that the patches could appear anywhere on the screen to encourage attention to the entire display. The auditory background noise stream was constructed from the discrete Fourier transform of an audio sample ( $48 \mathrm{kHz}$, stereo) of one TMS click. To this end, the phase of each resulting frequency bin was first randomly shuffled. The phase-shuffled frequency domain data were then back-transformed to the time domain using an inverse Fourier transform. Finally, these phase-shuffled TMS click sounds were randomly concatenated, resulting in a constant stream of noise, which contained the same frequency band power as the TMS clicks and was therefore particularly suited to attenuate the sound from the actual TMS pulses. Auditory targets consisted of transient fluctuations in noise volume for $500 \mathrm{~ms}$ at a $30 \mathrm{~Hz}$ rate (Fig. $1 B$ ). In other words, the volume of the noise was reduced and increased 30 times per second. Magnitudes of contrast (visual targets relative to noise) and volume (auditory targets relative to noise) were titrated individually in the intake session to $80 \%$ detection accuracy. Titration was performed using an adaptive staircase procedure following a one-up, three-down staircase in which three consecutive hits resulted in an increase in difficulty. The step-size of the change in difficulty decreased over trials converging to a level of $80 \%$ detection accuracy in 40 trials. The task was presented using MATLAB2012b (The MathWorks) and the Psychophysics Toolbox (http://psychtoolbox.org). Blocks were of varying length (range $=15-60 \mathrm{~s} ;$ mean $=37 \mathrm{~s} ; \mathrm{SD}=13 \mathrm{~s}$ ), unpredictable in duration for the participant, and were alternated between visual and auditory attention. The number of blocks per condition varied per subject as the length of each block was varied randomly (mean $=86$ blocks; $\mathrm{SD}=4$ blocks). The order of trials and blocks was generated before onset of the experiment such that an equal number of blocks were presented for both attentional conditions. A visual or auditory stimulus could appear every $2-22 \mathrm{~s}$ with a $50 \%$ chance of appearing in the attended modality. Participants were instructed to ignore stimuli in the unattended modality. In total, 48 auditory ( 24 targets) and 48 visual (targets) stimuli were presented. In total, 48 auditory and 48 visual stimuli were presented.

TMS. TMS was applied with biphasic pulse configuration using a MagVenture C-B60 Butterfly coil connected to a MagPro-X100 stimulator (MagVenture). The coil position and orientation were kept constant during the experiment using a frameless stereotactic neuronavigation system (Localite TMS Navigator) after coregistration of individual MRI scans. Coregistration was achieved (using the Localite software) in a two-step procedure by first marking the position of three anatomical 
A

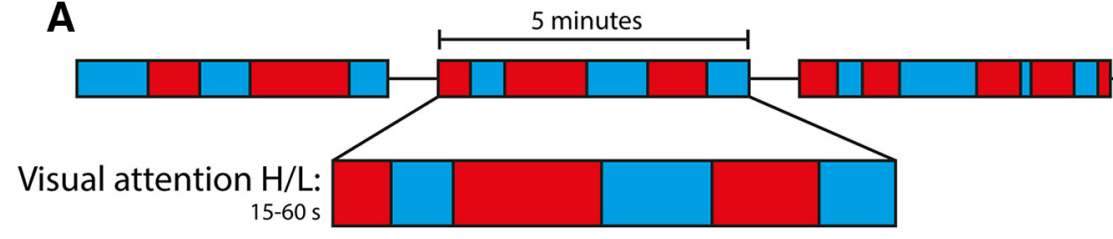
A/V Stimuli:

TMS/sham 1.5-2.5 s

C

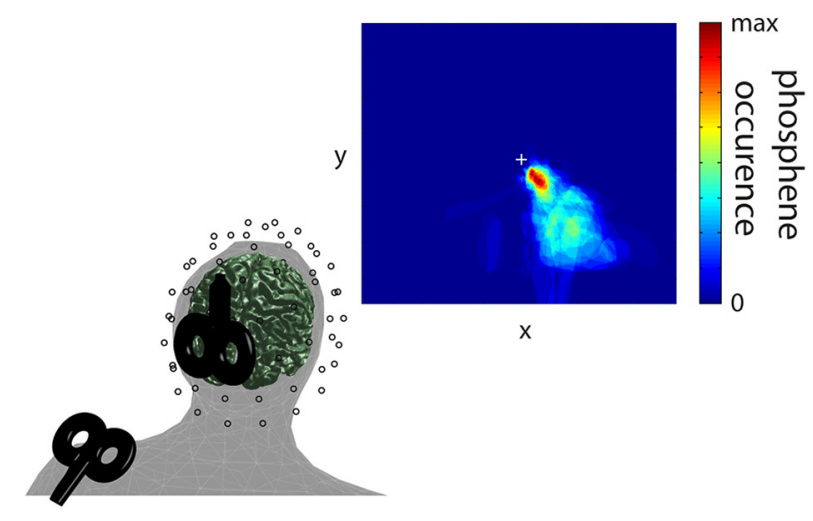

D

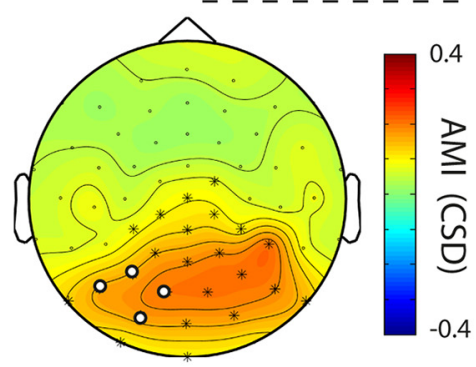

E

4
B

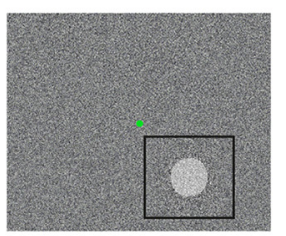

96 Auditory - (1) 药

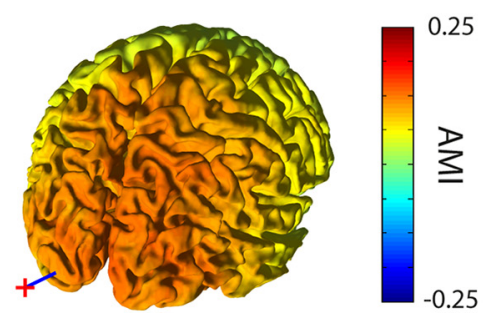

Figure 1. Experimental paradigm and setup. A, Experimental paradigm. Each run of 5 min consisted of "attend auditory" or "attend visual" blocks of varying length. Before the onset of each block, an audiovisual cue ("Look" or "Listen") indicated whether subjects had to attend to the visual modality (High visual attention) or to the auditory modality (Low visual attention). Both target (attended modality) and distractor (unattended modality) stimuli (500 ms duration) appeared unpredictably at a very low rate forcing participants to attend throughout the block. TMS and Sham pulses (shoulder TMS) were delivered randomly intermingled independently of the attentional task. $\boldsymbol{B}$, Participants had to maintain fixation while detecting near-threshold changes in simultaneously ongoing streams of auditory and visual background noise. Visual target stimuli were brief transient increases in contrast within a circular patch at an unpredictable location within $4^{\circ}$ to maximally $13^{\circ}$ visual angle around the fixation dot. Auditory target stimuli were brief fluctuations in noise volume. C, Site of stimulation and anatomical coregistration. EEG electrode and TMS-coil positions were coregistered to individual MRIs. The TMS target site was determined by a phosphene tracing procedure within the left visual cortex resulting in the depicted average phosphene probability map (note that stimulation intensity during the experiment was below phosphene threshold). The Sham site at the left shoulder was matched with regard to subjective auditory and somatosensory perception to control for multisensory evoked EEG responses. D, CSD topographical plot of the AMI: Low - High visual attention)/(Low + High visual attention). Channels indicated in white were used for analyses. Channels indicated in white and channels indicated with an asterisk showed significant alpha modulation at $p<0.001$ (corrected for False Discovery Rate) (Benjamini and Hochberg, 1995). $\boldsymbol{E}$, Source analysis of the AMI. Red cross and blue line indicate position of the TMS coil and target, respectively.

locations on the participant's head with a digitizing pen: nasion, left, and right outer canthus corresponding to markers set in the individual's MRI. Second, a scalp surface registration was performed to further improve the coregistration by tracing the scalp surface using a digitizing pen followed by a fitting of the individual MRI to the traced scalp surface. The coregistration was accepted if the root mean squared error of the fitting procedure was $<3 \mathrm{~mm}$.

The coil position, orientation, and stimulation intensity were individually determined in the intake session using a phosphene thresholding procedure (Dugué et al., 2011). During this procedure, the room was darkened and subjects adapted to darkness for $\sim 10 \mathrm{~min}$. Starting $2 \mathrm{~cm}$ left of the inion with the handle pointing upward, trains of 7 pulses at 20 $\mathrm{Hz}$ were applied at $70 \%$ maximum stimulator output (MSO) with the most significant induced current (i.e., first flank of second half wave) in the brain tissue flowing in the anterior-posterior direction. The coil was moved around in small steps until a location was found that reliably produced a phosphene in the lower right visual field. This procedure ensured that we effectively targeted the visual cortex. After a location was found, five trains of pulses were given after which subjects were asked to draw the outline of the phosphene percept with a mouse on the screen (Fig. $1 C$ provides a map for the phosphenes across subjects). Following this procedure, we assessed the intensity at which subjects would perce- ive phosphenes in $50 \%$ of trials (phosphene threshold) with single-pulse TMS using a manual staircase procedure. Starting at 70\% MSO, the intensity was increased in steps of 5\% per TMS pulse until the participant reported seeing a phosphene. The intensity was then decreased in steps of $1 \%$ until no phosphene was perceived anymore. Six pulses were applied to assess whether phosphenes were perceived in 50\% of trials. Depending on whether a phosphene was perceived in $>50 \%$ or $<50 \%$ of trials, the intensity was decreased or increased by $1 \%$, respectively, until the $50 \%$ threshold was found. To ensure the determined phosphene threshold was not confounded by participants' expectancy due to the regularity of the staircase procedure, we always pretended to change the intensity of the 6 consecutive pulses while actually keeping it constant. On average, phosphene threshold was $68 \%$ (SD $=8 \%$ ) of the MSO. Stimulation intensity during the experiment was set at $80 \%$ of phosphene threshold $($ mean $=55 \% \mathrm{MSO}, \mathrm{SD}=6 \% \mathrm{MSO})$.

Because auditory stimulation alone can cause phase reset in the visual cortex (Romei et al., 2012), a multisensory Sham condition served as control. To reproduce the auditory and somatosensory sensation associated with the TMS pulse without stimulating the brain, a second TMS coil (Sham) was placed on the left shoulder blade on the superior border of the scapula (Fig. 1C). For the Sham coil, stimulation intensity was matched to occipital TMS in terms of equal subjective loudness and 
reported sensory perception ( mean $=59 \% \mathrm{MSO}, \mathrm{SD}=8 \% \mathrm{MSO}$ ). Superimposed on the phase-shuffled TMS sound noise, we also presented a stream of randomly and densely packed ( 50 pulses per second) TMS click samples to further mask the sound of the actual TMS and Sham clicks.

Throughout the experiment, participants received 400 TMS and 400 Sham pulses per attention condition, randomly intermingled with an interpulse interval uniformly jittered between 1.5 and $2.5 \mathrm{~s}$, resulting in a total of 800 TMS and 800 Sham pulses.

MRI data acquisition. A high-resolution T1-weighted image $(\mathrm{TR}=$ $2250 \mathrm{~ms}, \mathrm{TE}=2.58 \mathrm{~ms}$, flip angle $=15^{\circ}, 208$ sagittal slices, in plane voxel size $=1 \times 1 \times 1 \mathrm{~mm}$, FOV $=224 \times 224 \mathrm{~mm}$ ) was acquired using a $1.5 \mathrm{~T}$ Avanto MRI scanner (Siemens) for TMS neuronavigation and EEG source analysis.

EEG data acquisition. The EEG was recorded from 61 channels and digitized at $5 \mathrm{kHz}$ (filter: $\mathrm{DC}$ to $1000 \mathrm{~Hz}$ ) with $0.1 \mu \mathrm{V} / \mathrm{bit}$ resolution using two battery-driven 32-channel BrainAmp DC amplifiers (BrainProducts) connected to a custom equidistant electrode cap (EasyCap M10) with TMS-compatible, extra flat $\mathrm{Ag}$ - $\mathrm{AgCl}$ ring electrodes with a slit in the ring to avoid magneto-induction (TMS Multitrodes, EasyCap). Skin resistance was kept $<5 \mathrm{kOhm}$ by thorough preparation using abrasive Abralyt $\mathrm{HiCl}$ electrode paste (EasyCap). Recording reference was at the vertex while a separate ground electrode was placed on the right collarbone. Electrode positions were digitized and coregistered to the individual anatomical MRI using a frameless stereotactic neuronavigation system (TMS Navigator, Localite).

EEG data analysis. EEG data were analyzed using MATLAB2014a and the FieldTrip MATLAB toolbox (The MathWorks) (http://www. fieldtriptoolbox.org) (Oostenveld et al., 2011). EEG data were preprocessed blind to the experimental conditions. First, EEG data were rereferenced to the common grand average of all EEG channels and epoched to -1.5 to $2.5 \mathrm{~s}$ intervals around the onset of the TMS pulse. (This time window extended into preceding and following trials and was chosen for the purpose of filtering and time-frequency analysis only. Later this time window was shortened to -0.2 to $1 \mathrm{~s}$ around the onset of TMS). The following steps are specific to TMS-EEG datasets and should be performed before any other forms of processing to avoid introducing additional artifacts. We have included a schematic pipeline (Fig. $2 A$ ) to aid researchers in dealing with TMS-EEG datasets. This pipeline has been worked out in detail on a different dataset presented as a tutorial on http://www.fieldtriptoolbox.org/tutorial/tms-eeg. A period of -1 to 7 ms relative to the TMS pulse was cut out (Fig. 2D) and excluded from the following steps in the artifact removal process to remove the initial "ringing artifact" (0-7 ms) resulting from the step response of the hardware filter of the EEG amplifier (Fig. $2 B, D$, red line). Pre-"ringing" and post"ringing" epochs were subsequently subjected to an independent component analysis (FastICA) to remove components reflecting (1) the "exponential decay artifact" (Fig. $2 F$ ) caused by electrode movements due to contraction of underlying muscles possibly in combination with concomitant depolarization of the electrode-electrolyte-skin interface (Ilmoniemi and Kicić, 2010); (2) residual muscle artifacts (Korhonen et al., 2011); and (3) to clean the data from eye-blinks, eye movements, line noise, and other muscle artifacts unrelated to TMS (Jung et al., 2000). The "exponential decay artifact" was identified in the ICA components after time-locking to the TMS pulse (Fig. 2E). All ICA components were removed that clearly showed an exponential decay and or a sinusoidal waveform (single cycle upwards of $67 \mathrm{~Hz}$, ending at $15 \mathrm{~ms}$; see, e.g., Fig. $2 C$ ), reflecting the muscle artifact starting at the TMS pulse and a topography that corresponded to the site of stimulation (Fig. $2 F$ ). On average, 12 of 60 components ( $12 \pm 2.5$, mean $\pm \mathrm{SD}$ ) were rejected, of which $1-2$ $(1.75 \pm 0.75)$ were related to the exponential decay and/or muscle artifact, $2-3(2.45 \pm 0.88)$ related to eye artifacts (blinks/saccades), $1-2$ $(1.83 \pm 0.76)$ related to line noise, and $6(6.08 \pm 2.20)$ related to other muscle artifacts unrelated to TMS. As ICA was not always able to fully capture the sinusoidal muscle artifact, cubic interpolation was used subsequently to replace the -1 to $15 \mathrm{~ms}$ period of the ICA-cleaned signal in all subjects (for a TMS-locked average cleaned solely of TMS-related artifacts, see Fig. $2 G$ ). Following TMS-artifact removal, the data were downsampled to $1 \mathrm{kHz}$. Before downsampling, a two-pass fourth-order Butterworth low-pass filter with a cutoff frequency of $200 \mathrm{~Hz}$ was applied to prevent aliasing. Subsequently, the data were $z$-transformed with the mean and SD calculated over all times and trials. Trials containing values deviating $>5$ SDs were removed. Visual inspection was then performed on the remaining trials to remove trials containing residual muscle contractions. Additionally, trials that contained visual or auditory target stimuli were removed. This resulted in rejection of on average 418 $(26 \%) \pm 23(2 \%)$ trials of which 96 trials due to the presence of an auditory or visual stimulus. After trial rejection, 296 trials remained per condition, on average. Subsequently, data were bandpass filtered between 3 and $45 \mathrm{~Hz}$ using a two-pass fourth-order Butterworth filter (104 $\mathrm{dB} /$ octave). Filtering was applied to the initial epochs ( -1.5 to $2.5 \mathrm{~s})$, but only the -0.2 to $1.0 \mathrm{~s}$ interval around the TMS pulse was considered for later analyses. The cutoff frequency of the high-pass filter was chosen relatively high to suppress contributions of slow fluctuations to the variance of faster components.

TMS-locked oscillations were analyzed based on time-frequency representations (TFRs) of power of the averaged TEPs by means of Fast Fourier Transform (FFT). The TEPs were multiplied with a Hanning tapered sliding time window moving in steps of $20 \mathrm{~ms}$. The length of the time window varied with frequency $(\mathrm{T}=3$ cycles $=3 / \mathrm{f})$. The mean and linear trends of the trial were subtracted from each time window before the time-frequency analysis in case of residual offsets and trends after filtering (Luck, 2005). An absolute baseline correction was performed for each TFR by subtracting power estimates of the pre-TMS period ( -0.5 to $-0.2 \mathrm{~s}$, to avoid overlap with the onset of the TEP) from the post-TMS interval. This was to avoid non-phase-locked alpha activity surviving averaging from influencing the post-TMS power estimates.

To assess individual ability to modulate the power of alpha oscillations during cross-modal shifts of attention (i.e., Low vs High visual attention), oscillatory activity was estimated in the baseline interval at -0.5 to $-0.001 \mathrm{~s}$ relative to the TMS pulse by applying a fixed time window to avoid confounding by the evoked response when using a sliding window. An alpha modulation index (AMI) was calculated for the pre-TMS interval (see Fig. 1D) as follows:

$$
\frac{\text { Power }_{\text {Low visual attention }}-\text { Power }_{\text {High visual attention }}}{\text { Power }_{\text {Low visual attention }}+\text { Power }_{\text {High visual attention }}} .
$$

Where indicated, topographical plots of scalp current source density (CSD) were calculated by fitting fourth-order spherical splines (first 9 Legendre polynomials, $\lambda=1 \mathrm{e}-5$ ) to the data at the last stage before plotting surface maps for the TEPs (Perrin et al., 1989, 1990). CSD maps of power values were calculated by estimating the CSD on the individual trials before performing Fourier analysis.

To localize the source of the alpha modulation due to the crossmodal shifts in attention before TMS onset, we used a DICS beamformer approach (Gross et al., 2001). The DICS algorithm uses the CSD matrix from the data and a lead field matrix to calculate a spatial filter. The CSD was calculated from a $500 \mathrm{~ms}$ pre-TMS time period $(-0.5$ to $-0.001 \mathrm{~s})$ pooled across attention conditions by calculating the FFT centered at $8 \mathrm{~Hz}$ of the data multiplied by a Hanning taper. A realistic three-layer volume conduction model was constructed using the individual MRI using the boundary element method (Oostendorp et al., 1989). A grid with $1 \mathrm{~cm}^{2}$ resolution was created per individual, which was subsequently normalized to MNI space. The lead field was calculated for each point in this grid. The spatial filter was then used to estimate power distributions for the attention conditions separately averaged over trials. The source of the TMS-locked alpha oscillations was similarly estimated with a spatial filter calculated on the single trial data ( -0.5 to $1 \mathrm{~s})$ pooled over all conditions. The calculated spatial filter was then applied to the power estimates from the 0.4 to $0.8 \mathrm{~s}$ period of the TEP for all conditions separately. Subsequently, an absolute baseline correction $(-0.5 \mathrm{~s}$ to $-0.1 \mathrm{~s}$, to have an equal length of data for baseline and post-TMS period) was performed to account for any alpha power not-related to TMS surviving averaging. Similar to the sensor level data, an alpha modulation index was calculated for the source level data (see formula above). 


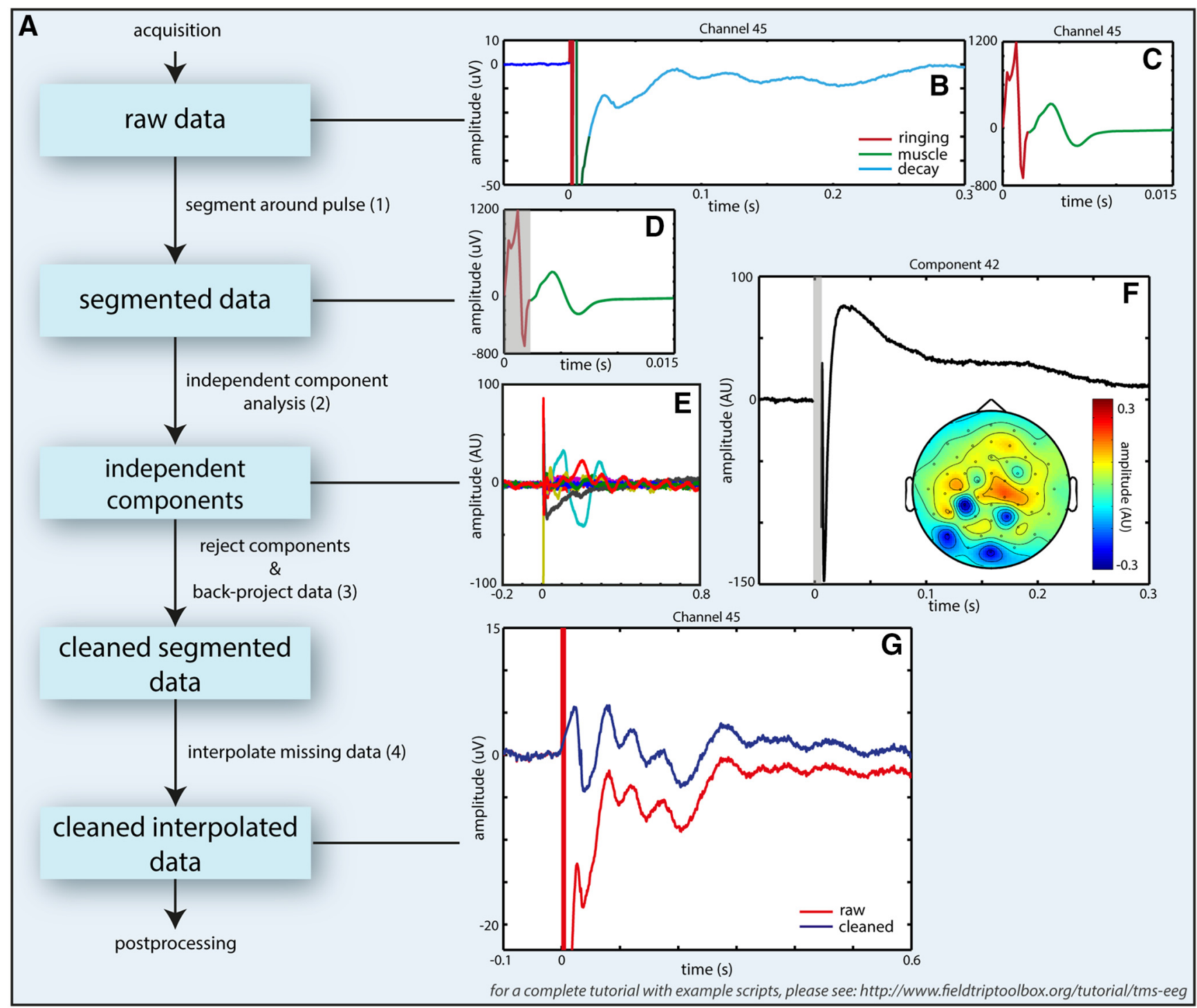

Figure 2. A, Processing pipeline for handling artifacts in TMS-EEG datasets. When dealing with TMS-EEG datasets, it is important to remove the TMS-related artifacts as early as possible in the processing pipeline (before any filtering preceding downsampling) to avoid introduction of additional "ringing artifacts" due to interaction of filter kernels with existing artifacts. First, the types and extent of artifacts were assessed from TMS-locked averages ( $\boldsymbol{B}, \boldsymbol{C})$. Colored lines indicate artifacts. The data were then (1) segmented to exclude the "initial ringing" artifact ( $\boldsymbol{B}, \boldsymbol{C}$, red line, $\boldsymbol{D}$, shaded area) before conducting an ICA (2). Time-locked averages of independent components were used to identify independent components capturing TMS-related artifacts ( $\boldsymbol{E}, \boldsymbol{F})$, taking into account topographical representations with extrema close to the stimulation site and adjacent cranial muscles. At this stage, other components related to non-TMS artifacts were identified as well. Then the data were back-projected to channel space (3) without the artifactual components. At this stage, the gap around the TMS pulse was interpolated. If the muscle artifact was not removed completely, this period was interpolated as well. TMS-locked averages were inspected afterward to check that the cleaning was successful (G). (For a tutorial with example scripts on how to deal with TMS-EEG datasets, see http://www.fieldtriptoolbox.org/tutorial/tms-eeg).

\section{Results}

To test whether TMS-locked alpha oscillations in the visual cortex reflect the same neuronal processes as spontaneous alpha oscillations, we investigated whether they are modulated in a similar manner by endogenous top-down attentional control (i.e., decreased during high and increased during low visual visual attention) (Fig. $1 A, B$ ). To this end, we simultaneously applied either TMS to the visual cortex or Sham stimulation to the shoulder and recorded 61-channel EEG while participants attended to either the visual (High visual attention) or auditory (Low visual attention) modality.

\section{Behavior and attention manipulation}

Performance in the cross-modal attention task remained close to $80 \%$ : participants detected $84 \%($ SEM $=2 \%)$ of the attended auditory targets and $81 \%(\mathrm{SEM}=2 \%)$ of the attended visual targets. Performance did not differ between conditions $(p>0.2$; paired sample $t$ test).

The topographical distribution of the AMI in Figure $1 D$ clearly shows that top-down attention did indeed modulate preTMS alpha power as intended. Low visual (i.e., high auditory) attention compared with High visual (i.e., low auditory) attention produced relative alpha power increases over posterior regions. The increase included the four channels close to the stimulation site $\left(t_{(23)}=2.35, p=0.0137\right.$, one-sided). Furthermore, source analysis allowed us to identify cross-modal alpha power modulation to bilateral visual and parietal cortices (Fig. $1 E)$. We could not observe a clear alpha power modulation in auditory cortices, which may be due to difficulties in localizing alpha activity from audio cortices (Frey et al., 2014). 
A
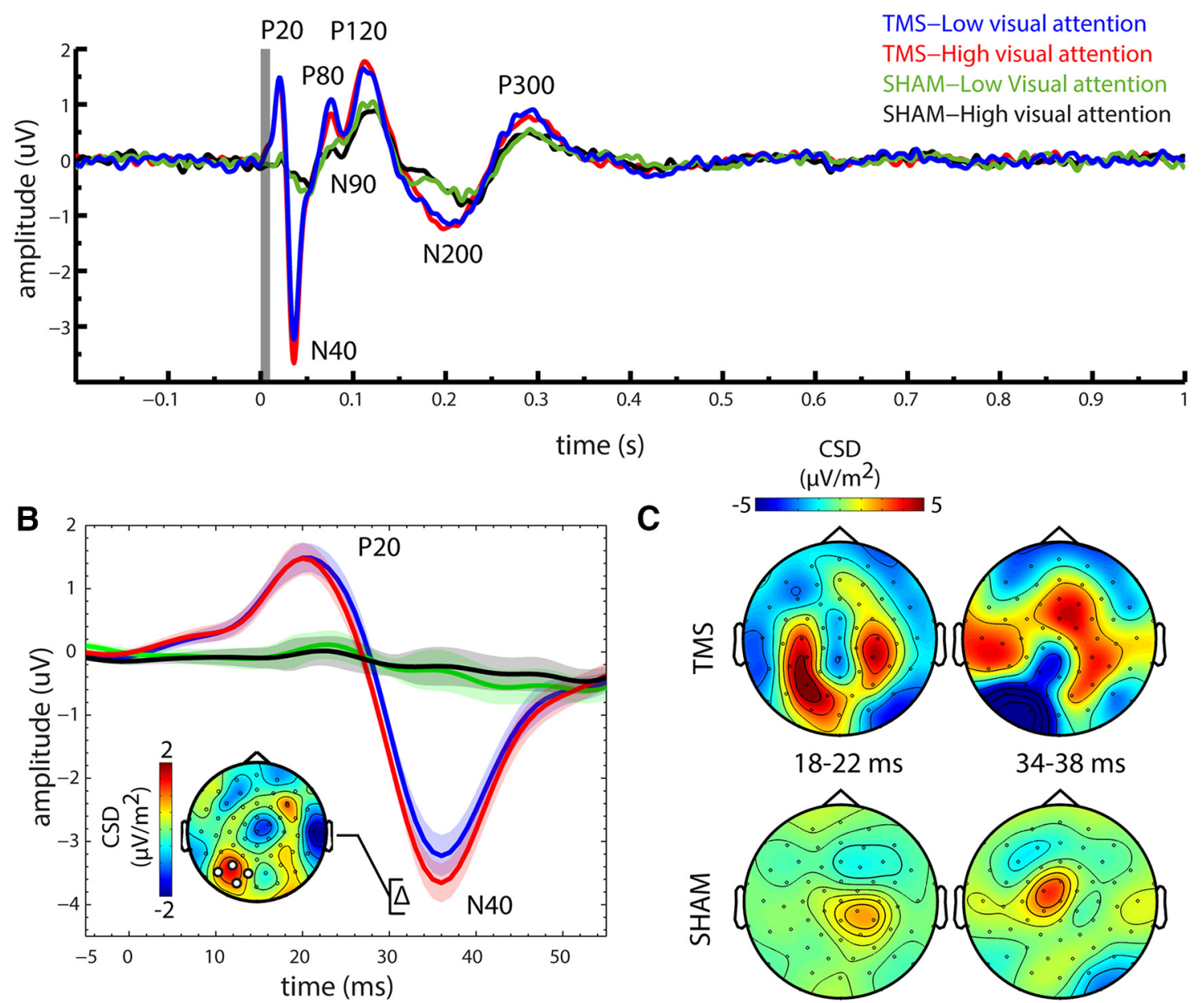

C
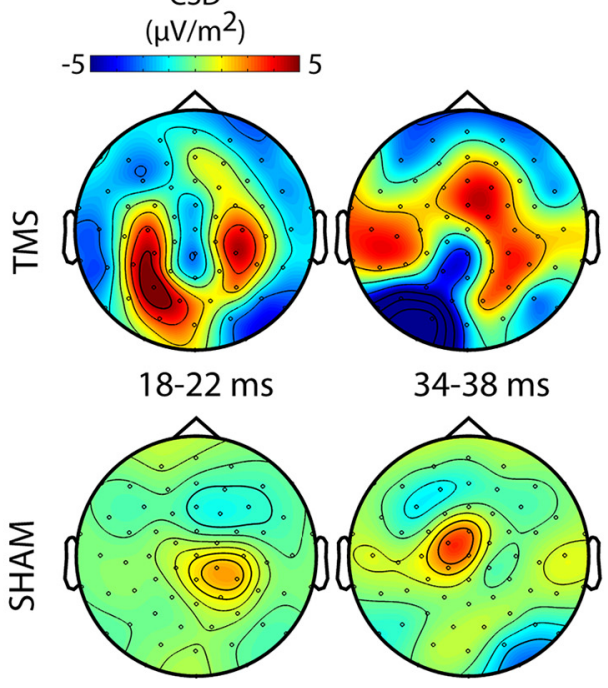

Figure 3. Attention modulated TEPS. A, TEPs (average of the four channels at the site of stimulation) are shown for TMS and Sham and separately for the High and Low visual attention conditions. B, Magnified view of the P20 and N40 TEP components for all conditions. Shaded areas represent the within-subject SEM (Loftus and Masson, 1994). Inset, Topographical plot of the difference between Low and High visual attention for TMS, highlighting the increased early N40 amplitude for High versus Low visual attention. C, Topographical plots (CSD) are shown for early TEP components, separately for TMS and Sham, but averaged across attention conditions, revealing clear differences in topography for TMS and Sham.

\section{Visual attention enhanced early TEP N40 component}

If top-down visual attention gates stimulus processing by upregulation and downregulation of visual cortex excitability (Gilbert and Li, 2013), this should be reflected by the immediate responsiveness of the visual cortex to transcranial stimulation (i.e., larger early evoked potentials during epochs of High compared with Low visual attention). Figure $3 A$ (inset) shows the TEPs for all experimental conditions for the four channels closest to the TMS coil.

TEPs were calculated by averaging trials time-locked to the TMS pulse separately for each condition. Unless specified otherwise, data were averaged from electrodes closest to the stimulation site (channel no. 28, 29, 44, 45; see Fig. $1 D$, approximately corresponding to PO8, P3, O1, and PO7 in the 10-20 system) and baseline corrected $(-0.05$ to $-0.01 \mathrm{~s})$. We observed seven clearly identifiable components (i.e., P20, N40, P80, N90, P120, N200, and P300) (Fig. 3A; other components may be prominent at other channels). Amplitudes of TEP components were extracted individually from the largest peak or trough, respectively, in the following time windows relative to the TMS pulse: 15-25, 25-45, 70-85, 85-110, 110-120, 150-250, and 250-350 ms. All components (i.e., P80-P300) after $50 \mathrm{~ms}$ were present not only for TMS but also Sham (albeit to a lesser degree; Fig. $3 A$ ), sug- gesting that a considerable part of the TEP may indeed be superimposed with multisensory evoked potentials related to the auditory and somatosensory stimulation. The early N40 component, a large negative deflection over the left visual cortex (Fig. $3 B, C)$, could only be observed during TMS, but not during Sham. Indeed, the deflection was significantly larger for TMS than for Sham (main effect of stimulation; $F_{(1,22)}=32.21, p=$ $\left.9 \times 10^{-6}\right)$. In addition, there was a significant interaction between stimulation and visual attention $\left(F_{(1,22)}=12.84, p=\right.$ $0.002)$, as the TMS-evoked N40 component was significantly larger during High than Low visual attention $\left(t_{(23)}=3.41, p=\right.$ $0.0024)$, whereas there was no attentional modulation during this time interval for Sham $(p>0.2)$. Notably, the topography of the attentional modulation of the TMS-evoked N40 was clearly restricted to the stimulation site (Fig. $3 B$, inset). An earlier positive deflection, the P20, also observed at the site of stimulation was also visible for TMS only and was significantly larger for TMS than for Sham (main effect of stimulation; $F_{(1,22)}=16.09, p=$ $5 \times 10^{-4}$ ); however, it was not modulated by visual attention (no main effect of visual attention; $p>0.2$; no interaction effect; $p>$ 0.7). For Sham (but not for TMS), analysis of a central channel cluster (seven channels centered around $\mathrm{Cz}$ ) showed a negative deflection at $117 \mathrm{~ms}$, which was significantly larger for low than 
for high visual attention. The topography of the difference is commensurate either with the N100 component of the auditory or somatosensory evoked potential. Thus, either an imperfect masking of the Sham click sound or sensation of left shoulder stimulation during Sham may be responsible for this component. Importantly, this did not affect the time window of our TMSrelated findings, and no such difference around the N100 was observed for low-high visual attention for TMS. In summary, the P20 and N40 deflections of the TEP appeared for TMS only, and the N40 was significantly modulated by visual attention, being larger during High than during Low visual attention.

\section{Visual attention suppressed TMS-locked alpha power}

We hypothesized that, if TMS-locked alpha oscillations are generated by the same neuronal mechanisms as spontaneous alpha oscillations, TMS-locked occipital alpha power should be decreased by High but increased by Low visual attention. Because we assumed alpha oscillations to be phase-locked to the TMS pulse, we calculated TFRs of power for the individually averaged TEPs. Unless specified otherwise, data were averaged from electrodes closest to the stimulation site (channel no. 28, 29, 44, 45; Fig. 1D). To avoid any potential confounds by multisensory (audio-tactile) evoked potentials within the first $400 \mathrm{~ms}$, TMSlocked alpha power was compared between experimental conditions in the window $0.4-1.0 \mathrm{~s}$ after stimulus between 8 and $12 \mathrm{~Hz}$. The TFRs were calculated per subject and averaged. No overlap with pre-TMS intervals or with the removed TMS pulse occurred. Based on the TFR for TMS pooled over Low and High visual attention, we determined the TMS-locked alpha power to be centered at $8 \mathrm{~Hz}$ and tapered off toward $0.8 \mathrm{~s}$, which determined the upper bound of our analysis window. Because of spectral leakage depending on the characteristics of the FFT, contributions of neighboring frequencies will leak into the estimation at $8 \mathrm{~Hz}$. Given the frequency resolution due to the length of the sliding time window at $8 \mathrm{~Hz}$ ( 3 cycles, or $375 \mathrm{~ms}$ ) and the use of a Hanning taper, estimates at $8 \mathrm{~Hz}$ will be biased toward contributions from frequencies at $8 \mathrm{~Hz} \pm 1.33 \mathrm{~Hz}$. In accordance with our hypothesis, topographical representations of TMS-locked alpha power (centered at $8 \mathrm{~Hz}$ ) in the $0.4-0.8 \mathrm{~s}$ post-TMS interval (i.e., after the early evoked potentials) show a clear attentional modulation: Low compared with High visual attention caused a local increase in TMS-locked alpha power at the site of stimulation $\left(t_{(23)}=1.87, p=0.037\right.$, one-sided $t$ test; Fig. $\left.4 A\right)$. This modulation did not occur for the Sham condition ( $p>0.6$; Fig. $4 B$ ). Importantly, this effect was corroborated by the directed interaction contrast $\left(\mathrm{TMS}_{\text {Low-High }}-\mathrm{Sham}_{\text {Low-High }}\right)$ comparing attentional modulation of TMS- and Sham-locked alpha power $\left(t_{(23)}=1.7317, p=0.048\right.$, one-sided $t$ test). TMS-locked alpha oscillations are not readily visible in the grand average TEP (Fig. $3 A$ ) probably due to phase cancellation over subjects. They were, however, observable in individual subjects. Figure $4 D$ shows TEPs of three representative subjects for whom TMS-locked alpha oscillations are clearly visible (low visual attention conditions) with voltage maps for one subject centered on a selection of peaks and troughs of the TMS-locked oscillation. The voltage maps highlight that the oscillating activity is strongest at the site of stimulation. Additionally, source analysis allowed us to identify the attention modulation of TMS-locked alpha activity to the left visual cortex overlapping both with the site of stimulation as well as the area in which alpha activity was modulated by attention before TMS onset (Fig. 4E).

To further quantify the TMS-locked alpha oscillation in the $0.4-0.8 \mathrm{~s}$ interval, we calculated the phase-locking factor (PLF) to assess intertrial phase coherence in the alpha band. Phaselocking analysis was performed using an equal amount of trials for each condition by random sampling, without replacement such that the amount of trials per condition was equated to the condition with the least amount of trials. As expected, TMS resulted in higher phase-locking than Sham $\left(F_{(1,23)}=7.79, p=\right.$ 0.01 ; Fig. $5 A)$. The PLF was not modulated by attention $\left(F_{(1,23)}=\right.$ $0.17, p=0.69)$. This is expected as attention mainly modulates power but not the phase of alpha oscillations (Mathewson et al., 2009, 2011). To verify that phase-locking was limited to the alpha-band, we calculated a time-frequency representation of the difference in phase-locking factor between TMS and Sham. We indeed found a time-frequency region of increased phaselocking in the alpha range, extending from the late TEP components and tapering off toward the end of the trial (Fig. $4 B$ ). Interestingly, we also found a decrease in phase-locking compared with Sham in the $\delta \theta$ band $(3 \mathrm{~Hz})$, starting $0.5 \mathrm{~s}$ after TMS onset. As this is not within the scope of the current study, we do not further discuss this observation.

In sum, these findings demonstrate that the amplitude of TMS-locked alpha oscillations is indeed modulated by visual attention the same way as spontaneous alpha oscillations are.

\section{Attentional modulation of spontaneous alpha power predicts attentional modulation of both TMS-locked alpha power and amplitude of the N40}

If TMS-locked alpha oscillations are actually reflecting a recruitment of the same neuronal mechanisms that constitute spontaneous alpha oscillations, the individual ability to modulate spontaneous alpha oscillations by visual attention should be predictive for the ability to modulate the TMS-locked alpha oscillations. Indeed, the normalized individual AMI $=($ High - Low visual attention $) /($ High + Low visual attention), at the site of stimulation during intervals directly preceding the TMS pulse, predicted the attentional modulation of TMS-locked alpha power (i.e., $\mathrm{TMS}_{\text {Low-High }}-\mathrm{Sham}_{\text {Low-High }}$ : Pearson $r_{(22)}=0.70$, $p=0.00014$ ) (Fig. 6A). Figure $6 B$ shows the topographical distribution of channel-wise correlation coefficients between pre-TMS AMI at a given channel and attentional modulation of TMSlocked alpha power (i.e., $\mathrm{TMS}_{\text {Low-High }}-$ Sham $_{\text {Low-High }}$ ) at the same channel. Notably, the relationship appears to be particularly strong at the site of stimulation (Fig. 6B). In short, the subjects for whom spontaneous visual alpha power was stronger modulated by top-down attention also were the subjects in which the TMS-locked alpha power was stronger modulated by attention. Interestingly, there was no such relationship between the attentional modulation of spontaneous alpha power and the strength of non-phase-locked alpha activity for the same timefrequency window $\left(r_{(23)}=0.17, p>0.4\right.$, Spearman correlation). Together with observed phase-locking analysis, this clearly shows that this relationship cannot be explained by a rebound of endogenous alpha activity into the state before TMS.

Taking into account the individual differences in attentional alpha power modulation at the stimulation site, we performed a $2 \times 2$ repeated-measures ANCOVA for TMS-locked alpha power with the factors visual attention (Low vs High) and stimulation (TMS vs Sham) and pre-TMS AMI as covariate. As shown in Figure $6 C$, the ANCOVA revealed a significant two-way interaction between visual attention and stimulation $\left(F_{(1,22)}=18.80\right.$, $p=0.00027)$, reflecting that visual attention modulated TMSbut not Sham-locked alpha power. Importantly, the magnitude of this attentional modulation effect on TMS-locked alpha power depended on the magnitude of attentional alpha power modula- 
A
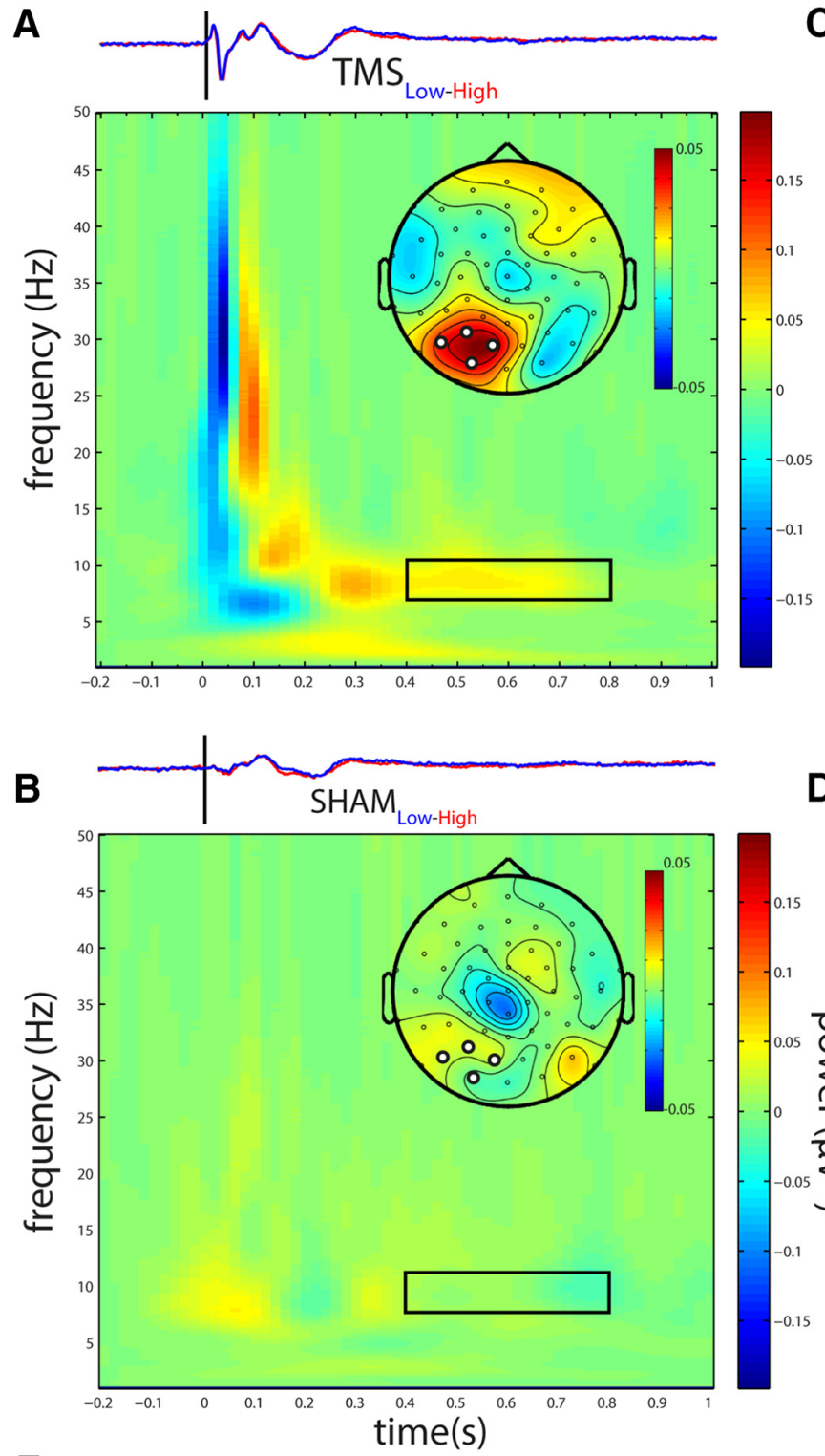

$\mathbf{E}$

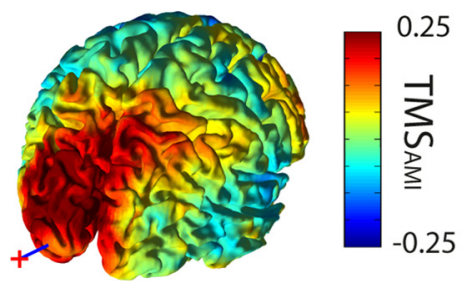

D
C
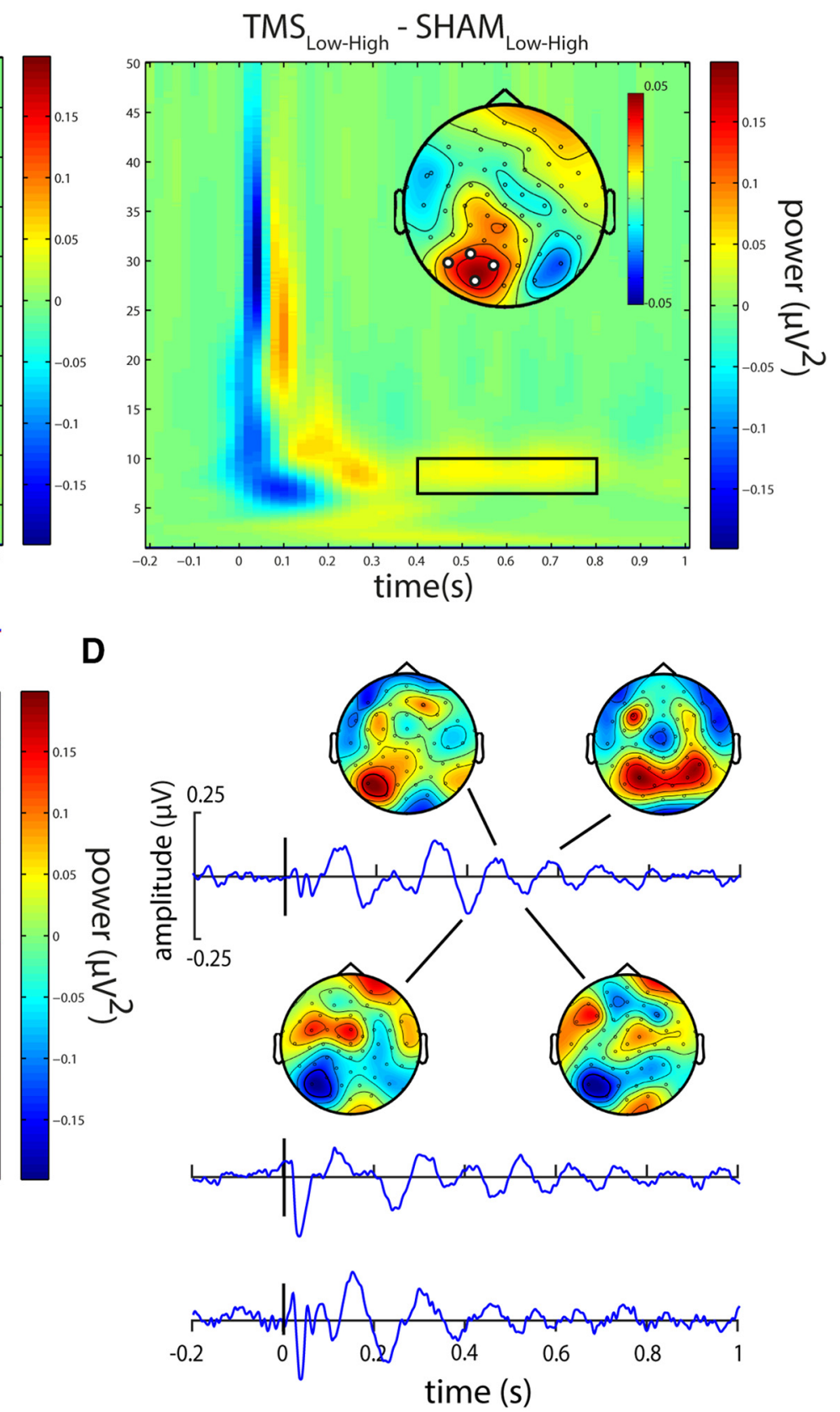

Figure 4. Attention modulates TMS-locked alpha power. A, TFRs show the attentional modulation (Low - High visual attention) of TMS-locked oscillatory power for TMS. $B$, Sham (bottom row). Topographical maps represent the average power from the time-frequency region of interest $(0.4-0.8 \mathrm{~s}$ after TMS, centered at $8 \mathrm{~Hz}$, FFT window size: $375 \mathrm{~ms})$ indicated by black rectangles within each TFR. The FFT window was multiplied with a Hanning taper, thereby biasing spectral estimation toward the center of the window. No overlap with pre-TMS intervals or with the removed TMS pulse occurred. The increased TMS-locked alpha power in the stimulated left visual cortex for TMS but not for Sham. For direct comparison with the TMS-locked power, TEPs separately for low (blue)

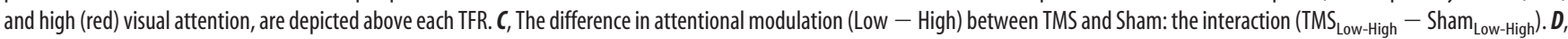
TEPs for three representative subjects in the Low visual attention condition. Individual TMS-locked alpha oscillations are clearly visible but not perfectly phase-aligned across subjects, thus averaging out at the group TEP. Topographical voltage maps for several half-waves of the TMS-locked alpha oscillation demonstrate an oscillatory pattern mainly restricted to the stimulated left visual cortex. E, Source analysis of the normalized $\left(\right.$ TMS $_{\text {Low }}-$ High $/ T M S_{\text {Low }}+$ High $)$ attention modulation of TMS-locked alpha power demonstrates the modulation of TMS-locked alpha power by attention to be localized to the visual cortex in the stimulated left hemisphere. Red cross and blue line indicate position of the TMS coil and target, respectively.

tion before TMS, as indicated by the highly significant three-way interaction between AMI, visual attention, and stimulation $\left(F_{(1,22)}=26.153, p=0.00004\right)$. That is, subjects that were better able to modulate spontaneous alpha power during pre-TMS intervals by visual attention also showed stronger attentional mod- ulation of the TMS-locked alpha power consistent with the correlation analysis in Figure 6A. Importantly, the results of the ANCOVA additionally show that attention has an effect on TMSlocked alpha activity over and above that of the pre-TMS alpha modulation, as this is corrected for in the two-way interaction. 
A

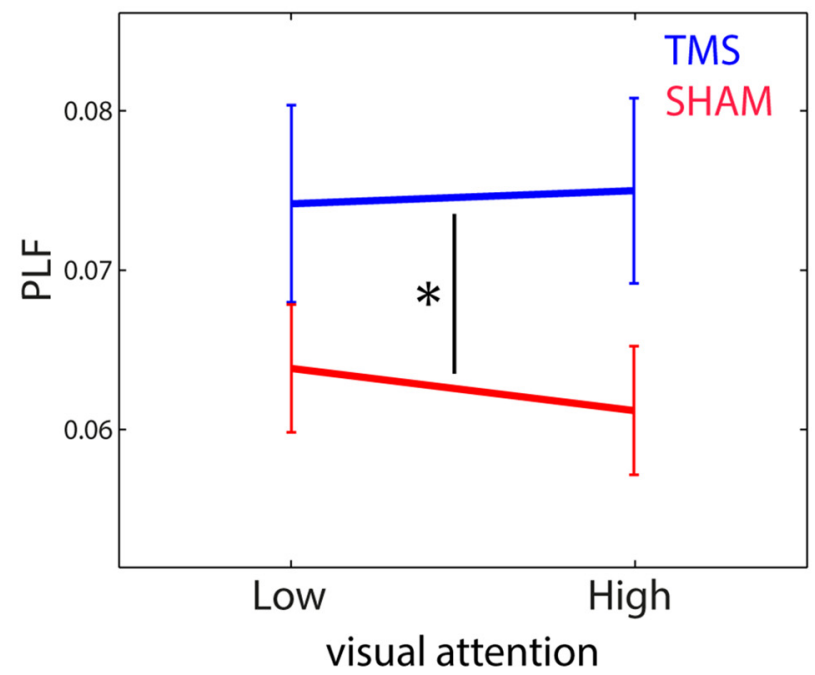

B

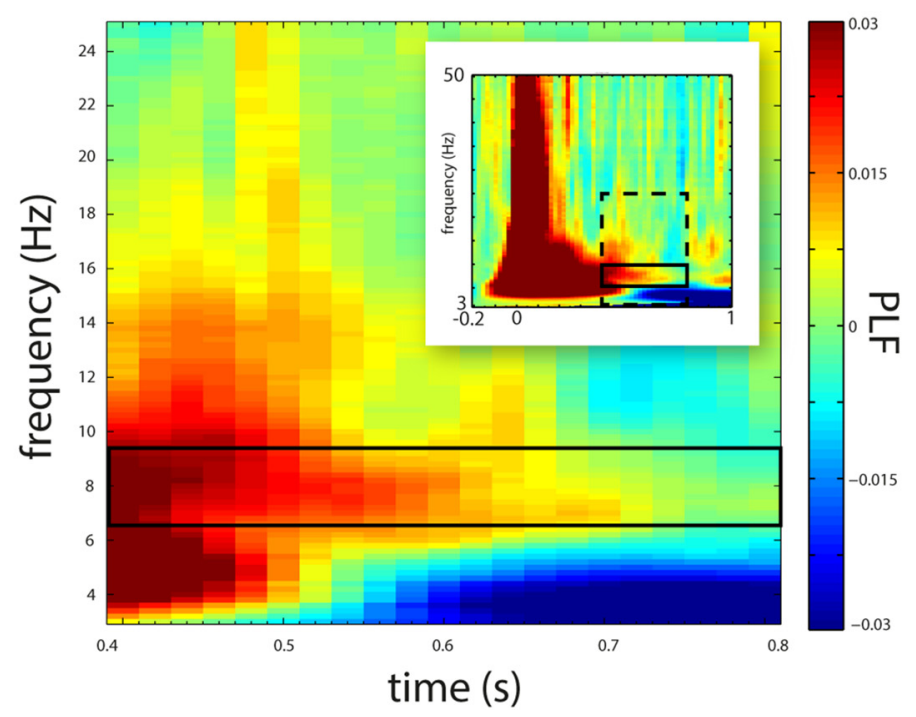

Figure 5. TMS caused increased phase-locking in the alpha band. $A$, Line plot indicating the PLF for the $0.4-0.8$ s alpha response (centered at $8 \mathrm{~Hz}$ ) to TMS (blue) and Sham (red), separately for both levels of visual attention (Low vs High). A $2 \times 2$ ANOVA of visual attention (Low vs High) and stimulation (TMS vs Sham) revealed a main effect of stimulation only. Error bars indicate SEM. $\boldsymbol{B}$, TFR of the PLF shows the comparison of TMS versus Sham, pooled over Low and High visual attention from $0.4-0.8 \mathrm{~s}$ for $3-25 \mathrm{~Hz}$ (dashed box in inset). Solid box represents the analysis window. Inset, TFR of PLF for the entire trial ( -0.2 to $1 \mathrm{~s})$ for $3-50 \mathrm{~Hz}$.

In addition, we assessed similarities in peak frequency. To maximize frequency resolution while keeping estimation similar across data segments, we estimated peak frequency in the alpha band from $0.4 \mathrm{~s}$ data segments (as this is the largest uncorrupted segment obtainable from the post-TMS interval) multiplied by a Hanning taper, zero-padded to $10 \mathrm{~s}$ (Fig. 7A). We found that the peak frequency of TMS-locked alpha correlated significantly with the peak frequency during the training session $\left(r_{(24)}=0.44, p=\right.$ 0.038 ; Fig. $7 B$ ). Whereas the peak frequency of TMS locked alpha did not correlate with the alpha frequency during the resting state measurement, or the pre-TMS interval, average peak frequency of the attentional modulation of TMS-locked alpha (9.34 \pm 0.49 $\mathrm{Hz}$ ) did not significantly differ from (1) spontaneous alpha frequency in the TMS-free training session $(9.47 \pm 0.41 \mathrm{~Hz}),(2)$ alpha frequency during the resting state measurement $(10.01 \pm$ $0.30 \mathrm{~Hz}$ ), and (3) alpha frequency in the pre-TMS interval $(10.11 \pm 0.29 \mathrm{~Hz}$ ) (paired $t$ tests; $p>0.4, p>0.1$, and $p>0.2$, respectively; Fig. $7 A$ ).

We also tested whether the magnitude of attentional modulation of the N40 TEP component was predicted by the extent to which attention did modulate spontaneous alpha power before TMS. We found that the interaction effect between stimulation and visual attention in the N40 was correlated with pre-TMS AMI $\left(r_{(22)}=0.65, p=5 \times 10^{-4}\right)$, demonstrating that the modulation of the N40 by attention was stronger in subjects who showed higher AMI before TMS (Fig. 6D).

To test whether it is pre-TMS alpha power itself, regardless of visual attention, that predicts TMS-locked alpha power, we pooled the data of both attention conditions and binned them into High and Low pre-TMS alpha power by means of a median split of all trials with respect to alpha power during the -0.5 to $-0.001 \mathrm{~s}$ interval, separately for TMS and Sham. The N40 component was indeed larger (more negative) when evoked during Low compared with High alpha power $\left(t_{(23)}=2.32, p=0.03\right.$ ) for TMS, but not for Sham $(p>0.7)$. Furthermore, a significant main effect of stimulation could be observed $\left(F_{(1,23)}=32.5, p=\right.$ $\left.8 \times 10^{-6}\right)$, but no significant interaction between stimulation and alpha power $(p>0.1)$. In contrast, TMS-locked alpha power was not significantly higher for High compared with Low alpha power trials, either for TMS ( $p>0.1$, one-sided paired $t$ test) or for Sham $(p>0.5$, paired $t$ test), although a directed interaction test did reveal a significant interaction between stimulation condition and level of pre-TMS alpha power $\left(t_{(23)}=2.14, p=0.04\right)$.

Together, these findings demonstrate that the individual ability to top-down modulate spontaneous alpha power by attention predicts the amount of attentional modulation of both TMSlocked alpha power and the N40 TEP component, but in opposite direction. Therefore, TMS-locked and spontaneous alpha oscillations are likely reflecting recruitment of the same neuronal mechanisms, whereas the N40 TEP component rather reflects cortical excitability.

\section{Discussion}

Single-pulse TMS (but not Sham) evoked an early TEP component (N40) as well as subsequent alpha-like oscillations (400$800 \mathrm{~ms}$ after TMS) that were both localized to the stimulated left visual cortex. Top-down visual attention increased the amplitude of the N40 TEP component but decreased the power of the TMSlocked alpha-like oscillation. Moreover, the extent to which attention modulated TMS-locked alpha power was predicted by the extent to which it modulated spontaneous alpha power in the stimulated cortical region during pre-TMS intervals. We thus provide new evidence that TMS-locked alpha responses rely on the same neuronal processes as spontaneous alpha activity.

\section{TMS-locked alpha oscillations "behave" like spontaneous alpha oscillations}

First and foremost, the power of TMS-locked alpha was topdown modulated by endogenous visual attention in the same way as the power of spontaneous alpha. As shown by our own (Fig. 1D) as well as previous work using an audio-visual crossmodal attention task (Adrian, 1944; Fu et al., 2001; Mazaheri et al., 2014), spontaneous alpha power in the visual cortex is increased during periods of low visual attention (when subjects 
A

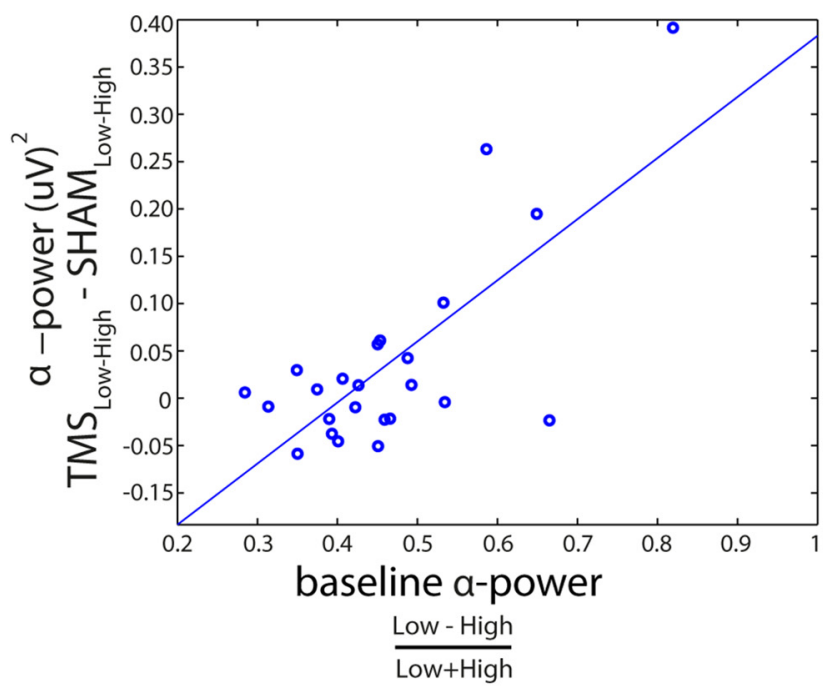

C

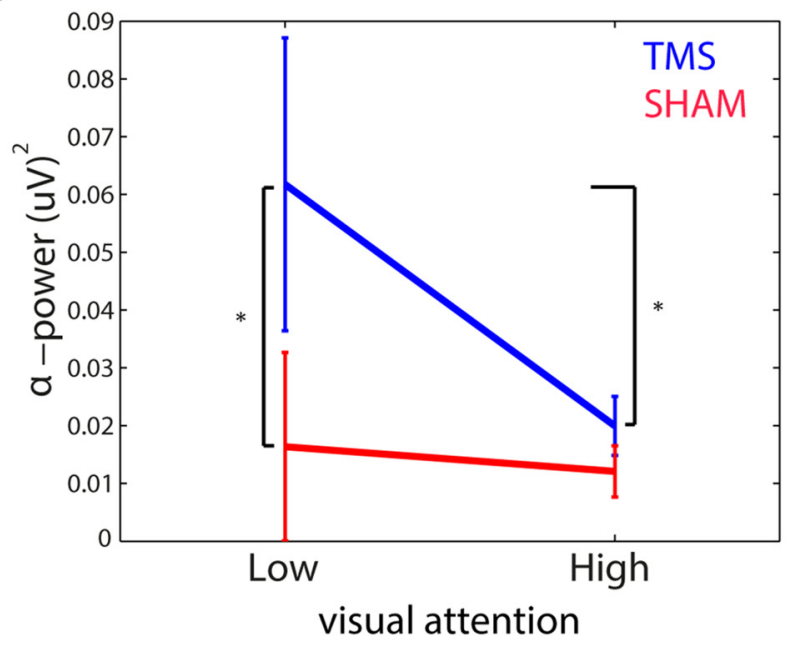

B
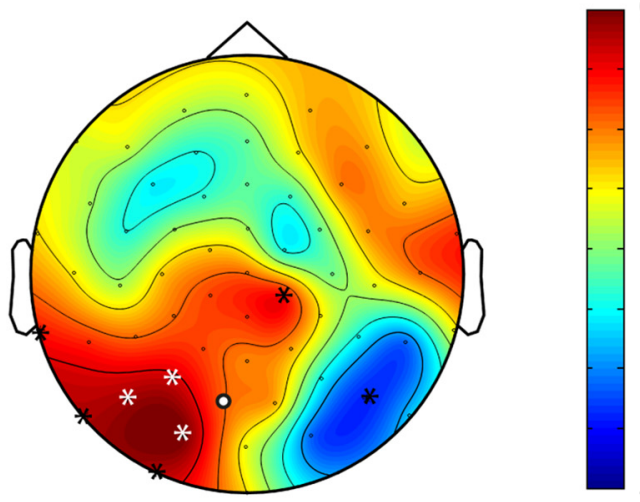

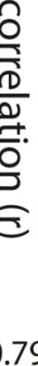

D

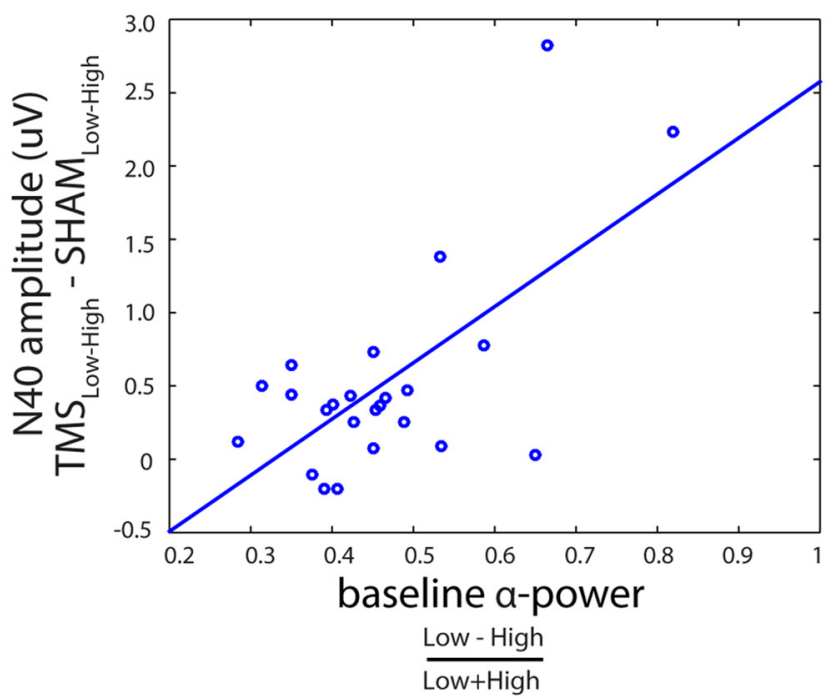

Figure 6. Impact of pre-TMS alpha visual attention modulation on TMS-locked alpha power modulation. $A$, Participant's ability to modulate spontaneous alpha at the stimulation site during pre-TMS intervals by top-down visual attention predicts the strength of attentional TMS-locked alpha power modulation at the stimulation site (i.e., white channels in $\boldsymbol{B}$ ). The differential modulation of TMS-locked alpha power by TMS and Sham (i.e., TMS Low-High $_{\text {- Sham }}$ Low-High) ) is predicted by AMI at pre-TMS baseline. $\boldsymbol{B}$, Topographical plot depicts all correlation coefficients between pre-TMS alpha power modulation at a given channel and the attentional modulation of TMS-locked alpha power (i.e., TMS $_{\text {Low-High }}$ - Sham ${ }_{\text {Low-High }}$ ) at the same channel. The better spontaneous alpha power could be modulated by top-down attention in the left (stimulated) visual cortex; the stronger also TMS-locked alpha power was modulated in that region by attention. Channels indicated by an asterisk showed a significant correlation ( $p<0.05$, corrected for False Discovery Rate) (Benjamini and Hochberg, 1995). Channels indicated by a white asterisk, or white dot with black outline, represent the channels used in the analyses throughout the study. C, Visual attention modulated TMS-locked alpha power for TMS but not for Sham. Asterisks indicate significant ( $p<0.05$ ) post hoc comparisons following significant interaction of the stimulation $\times$ visual attention ANCOVA using attentional AMI before TMS as covariate. $D$, Participant's ability to modulate spontaneous alpha at the stimulation site during pre-TMS intervals by top-down attention predicts the strength of the attentional modulation of the N40 TEP component. Because of the negative sign of the N40 component, positive here means a stronger negative deflection for High visual attention than for Low visual attention.

attend the auditory input stream) but decreased during periods of high visual attention (when subjects attend the visual input stream). This is thought to reflect the inhibitory nature of alpha oscillations, upregulated in task-irrelevant brain regions to suppress and downregulated in task-relevant regions to facilitate information processing (Klimesch et al., 2007; Jensen and Mazaheri, 2010). The TMS-locked alpha oscillation at 400-800 $\mathrm{ms}$ is not readily evident in the grand average TEP (Fig. $3 A$ ) because its onset and frequency (and thus phase) are coherent within but not across subjects (Fig. $4 D$ ), causing phase cancelation when averaging. However, grand averages of individual TEP time-frequency representations clearly reveal that TMS but not Sham evokes an alpha oscillation of several cycles in the an- alyzed post-ERP period from 400 to $800 \mathrm{~ms}$ (Fig. $4 A-C$ ). Importantly, phase-locking analysis confirmed that this alpha oscillatory response is phase-locked to the TMS pulse (stronger than for Sham; Fig. 5A) and thus not a mere TMS-related power modulation. For instance, it cannot be explained by a rebound of pre-TMS alpha after a TMS-induced alpha desynchronization (with alpha merely surviving averaging due to its high amplitude despite a lack of phase-locking). As for spontaneous alpha, endogenous attention modulated the power but not the phaselocking of TMS-locked alpha oscillations. The reason for TMSlocked alpha not being visible before $400 \mathrm{~ms}$ is unclear. Possibly, TMS elicited distinct episodes of excitation and suppression (Moliadze et al., 2003). Alternatively, TMS-locked alpha started 

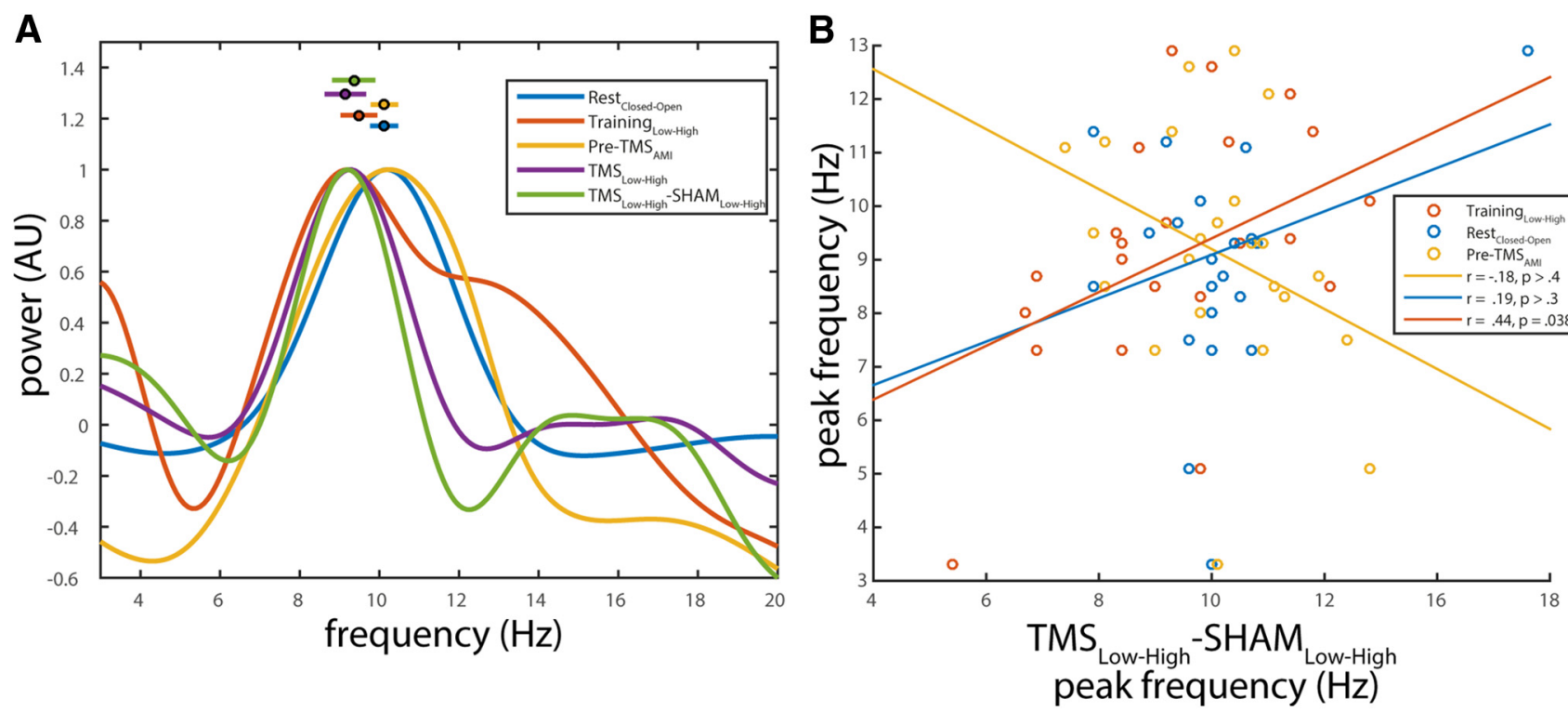

Figure 7. A, Average power spectra showing peak alpha frequency for all attentional contrasts. Each line indicates the normalized power spectrum (all values divided by maximum power within condition) for the attentional contrasts shown in the legend. Data points with error bars indicate mean \pm SEM individual peak frequencies for each contrast. $\boldsymbol{B}$, Peak frequency of attentionally modulated TMS-locked alpha correlates with peak frequency of attentionally modulated spontaneous alpha in the training session (but not in the main experiment or for eyes closed-open). Each circle represents the peak frequency of the attentionally modulated TMS-locked alpha ( $x$-axis) versus the peak frequency of attentionally modulated spontaneous alpha during training (gold) and pre-TMS intervals (blue), as well as of the closed-open eyes contrast at rest (orange). Lines indicate least-squares regression lines for the separate correlations (see figure legend for Pearson $r$ and $p$ values).

earlier but got masked by the TEPs and multisensory ERP components during the early post-TMS period. Indeed, TMS-locked alpha power seems to be modulated by attention already $250-$ $400 \mathrm{~ms}$ after TMS (Fig. 4A) but did not yet differ significantly from Sham during that period (Fig. $4 B$ ).

The second finding supporting the notion that TMS-locked alpha resembles spontaneous alpha comes from the interindividual correlation of their respective attentional modulation effects. The extent to which endogenous attention modulated TMS-locked alpha power was predicted by the amount of attentional modulation of spontaneous alpha power in the stimulated cortical region during pre-TMS intervals (Fig. 6A,B). These interindividual differences are possibly linked to structural differences in white matter tracts (Marshall et al., 2015). Two complementary interpretations are commensurate with this finding but cannot be disentangled based on the current experimental design. The first possibility is that TMS triggers a new alpha oscillation in the stimulated neuron population, which is independent of the global spontaneous alpha oscillation at the time of stimulation. This would characterize an evoked response in the classical sense. Yet, both oscillations would be top-down modulated to the same degree by visual attention according to the current task condition and depending on the subjects' individual ability. Alternatively, TMS may actually reset the ongoing spontaneous alpha oscillation, but only in the stimulated neuron population. Again, its amplitude modulation would directly depend on the amplitude modulation of the global spontaneous alpha oscillation that has been reset. This would explain the evoked potential as emerging from phase-resetting of a spontaneous oscillation. Interestingly, the size of TMS-locked alpha power could not be fully explained by pre-TMS alpha power. The size of the TMS-locked alpha response seems to rather depend on the strength of top-down attentional modulation of alpha power than on the mere level of spontaneous alpha power randomly fluctuating at the stimulation site.

The peak frequencies of the attentional modulation of TMS-locked and spontaneous alpha power during the TMS-free training session were comparable and even correlated across participants. The frequency of TMS-locked alpha power did not correlate with that of attention-modulated alpha power during pre-TMS intervals of the main task or during rest. Importantly, however, they did not differ significantly either. Thus, although attentional modulation of TMS-locked alpha power was at the lower boundary of the typical alpha band $(8-9 \mathrm{~Hz}$, depending on the method of estimation), it was well within the range of spontaneous alpha power modulation observed in our sample. Indeed, considerable interindividual but also intraindividual differences in peak alpha frequency have been reported, varying between subjects by an SD of $2.8 \mathrm{~Hz}$ and within subjects by an SD of $0.9 \mathrm{~Hz}$, interestingly increasing with task engagement (Haegens et al., 2014). It is thus possible that attentional modulation in the presence of TMS in the main task required more engagement than in the TMS-free training session. Although we found no significant differences between TMS-locked and spontaneous alpha in the current study, they are principally conceivable. While spontaneous alpha may be constantly driven by a neuronal generator (e.g., a thalamic pacemaker), a single TMS pulse may evoke transient resonance in the same circuits but slow down quickly due to progressive phase-desynchronization in the absence of repetitive synchronizing input. In summary, approximately comparable peak frequencies together with the correlation of peak frequencies for attentional modulation of TMS-locked alpha and spontaneous alpha during the training session provide additional evidence for common mechanistical grounds. Yet, the lack of correlation with the frequency of spontaneous alpha power modulation during the main task remains unexplained.

\section{TMS-locked alpha oscillations cannot be explained by multisensory stimulation artifacts}

The application of TMS is inevitably accompanied by multisensory stimulation. Auditory stimulation is caused by the typical "click" sound of the discharging TMS coil, somatosensory stim- 
ulation by the costimulation of cranial muscles and peripheral nerves in the skin, and visual perceptions (i.e., phosphenes) may be triggered when stimulating the visual cortex. All these inputs cause evoked potentials in the respective sensory systems, overlaying transcranially evoked brain potentials within the first $\sim 400 \mathrm{~ms}$. As demonstrated for auditory stimuli (Romei et al., 2012), they may even cause cross-modal phase resetting of alpha oscillations in the visual cortex, which can be mistaken for TMSlocked alpha oscillations. We prevented phosphene perception by adjusting stimulation intensity to $80 \%$ phosphene threshold. In addition, we designed a multisensory extracranial Sham stimulation controlling for audio-tactile input but lacking actual brain stimulation. Perfectly matching the subjective experience of cranial TMS is hardly possible. Nevertheless, stimulation of the ipsilateral shoulder blade, individually matched for auditory and somatosensory perception, at least produced a left-lateralized audio-tactile input of comparable intensity and resulted in a strikingly similar "TEP" (Fig. 3). Indeed, TMS and Sham TEPs shared most of their components (except the N40 and P20), albeit with slightly lower amplitude for Sham, emphasizing the necessity of appropriate control conditions in TMS-EEG research.

Importantly, we observed TMS-locked alpha oscillations as well as their attentional modulation only for visual cortex TMS but not for Sham, rendering it unlikely that these effects resulted from multisensory stimulation or cross-modal phase-resetting. Moreover, the effects we observed were restricted to the stimulated left visual cortex (Fig. 4) and emerged in the analyzed time interval of 400-800 ms (unaffected by multisensory ERPs), whereas Romei et al. (2012) observed more shorter-lasting crossmodal phase-resetting effects $(<300 \mathrm{~ms})$ throughout the entire visual cortex.

Together, we are confident that the observed TMS-locked alpha oscillation did actually result from transcranial stimulation of cortical neurons in the left visual cortex rather than any kind of accompanying sensory stimulation. If properly controlled, TMSEEG thus provides a suitable tool not only to study but also to manipulate cortical oscillations (e.g., via "entrainment").

\section{N40 reflects excitability of the visual cortex}

TMS to the visual cortex evoked a response consisting of several components (P20, N40, P80, N90, P120, N200, P300; Fig. 3). Although most components were visible for both TMS and Sham, and are thus most likely attributable to unspecific multisensory stimulation effects, the N40 component was evident for TMS only and was additionally modulated by attention. As expected for early TEP components, its amplitude was larger during periods of high compared with low visual attention, a pattern already known from the attentional amplification of visual evoked potentials (Rajagovindan and Ding, 2011). Interestingly, the N40 amplitude also correlated with the individual extent to which spontaneous alpha power was modulated by attention during pre-TMS intervals, thus paralleling the correlation with TMS-locked oscillations but in opposite direction. As a strong link has been established between pre-TMS alpha power and the level of visual cortex excitability (Thut et al., 2006; Romei et al., 2008a,b), the N40 amplitude appears to index the level of visual cortex excitability at the time of stimulation. This finding is in line with TMS-EEG work targeting the primary motor cortex, where the N40/45 was localized to the stimulation site and its amplitude correlated with stimulation intensity and conditioning pulse intensity in a paired-pulse design (Paus et al., 2001; Premoli et al., 2014). Also, during deep sleep, the amplitude of a positive component of comparable latency (P40) indexed motor cortical excitability fluctuations during the sleep slow oscillation (Bergmann et al., 2012). However, because the motor cortex N45 has been recently linked to GABA-A-ergic inhibition (Premoli et al., 2014), it may reflect the inhibitory feedback in response to TMS-induced cortical excitation (which nevertheless scales with the amount of excitation), rather than the initial excitation itself. Future research is needed to further evaluate the N40 component as an index for cortical excitability and inhibition.

In conclusion, our findings strongly suggest that TMS-locked alpha oscillations do indeed rely on the same neuronal mechanisms as spontaneous alpha oscillations. They are top-down modulated by endogenous attention in the same way and are tightly linked to the extent subjects are able to modulate spontaneous alpha power in the stimulated brain region. Importantly, these effects cannot be explained by mere multisensory stimulation as ruled out by comparison with a high-level audio-tactile Sham condition. However, it remains to be explained why the peak frequency of TMS-locked alpha did not perfectly match that during pre-TMS intervals. Conversely, the early N40 TEP component is also modulated by attention, but in the opposite direction, presumably indexing visual cortex excitability at the time of stimulation. Therefore, a local alpha response to single-pulse TMS, possibly reflecting phase-rest of spontaneous alpha, may be the basis for the effectiveness of transcranial entrainment protocols.

\section{References}

Adrian ED (1944) Brain rhythms. Nature 153:360-362. CrossRef

Benjamini Y, Hochberg Y (1995) Controlling the false discovery rate: a practical and powerful approach to multiple testing. J R Stat Soc B 289-300.

Bergmann TO, Mölle M, Schmidt MA, Lindner C, Marshall L, Born J, Siebner HR (2012) EEG-guided transcranial magnetic stimulation reveals rapid shifts in motor cortical excitability during the human sleep slow oscillation. J Neurosci 32:243-253. CrossRef Medline

Buzsáki G, Draguhn A (2004) Neuronal oscillations in cortical networks. Science 304:1926-1929. CrossRef Medline

Dugué L, Marque P, VanRullen R (2011) The phase of ongoing oscillations mediates the causal relation between brain excitation and visual perception. J Neurosci 31:11889-11893. CrossRef Medline

Engel AK, Fries P (2010) Beta-band oscillations: signalling the status quo? Curr Opin Neurobiol 20:156-165. CrossRef Medline

Frey JN, Mainy N, Lachaux JP, Muller N, Bertrand O, Weisz N (2014) Selective modulation of auditory cortical alpha activity in an audiovisual spatial attention task. J Neurosci 34:6634-6639. CrossRef Medline

Fries P (2005) A mechanism for cognitive dynamics: neuronal communication through neuronal coherence. Trends Cogn Sci 9:474-480. CrossRef Medline

Fu KM, Foxe JJ, Murray MM, Higgins BA, Javitt DC, Schroeder CE (2001) Attention-dependent suppression of distracter visual input can be crossmodally cued as indexed by anticipatory parieto-occipital alpha-band oscillations. Brain Res Cogn Brain Res 12:145-152. CrossRef Medline

Garcia JO, Grossman ED, Srinivasan R (2011) Evoked potentials in largescale cortical networks elicited by TMS of the visual cortex. J Neurophysiol 106:1734-1746. CrossRef Medline

Gross J, Kujala J, Hamalainen M, Timmermann L, Schnitzler A, Salmelin R (2001) Dynamic imaging of coherent sources: Studying neural interactions in the human brain. Proc Natl Acad Sci U S A 98:694-699. CrossRef Medline

Gilbert CD, Li W (2013) Top-down influences on visual processing. Nat Rev Neurosci 14:350-363. CrossRef Medline

Haegens S, Luther L, Jensen O (2012) Somatosensory anticipatory alpha activity increases to suppress distracting input. J Cogn Neurosci 24:677685. CrossRef Medline

Haegens S, Cousijn H, Wallis G, Harrison PJ, Nobre AC (2014) Inter- and intra-individual variability in alpha peak frequency. Neuroimage 92: 46-55. CrossRef Medline

Hanslmayr S, Gross J, Klimesch W, Shapiro KL (2011) The role of alpha oscillations in temporal attention. Brain Res Rev 67:331-343. CrossRef Medline 
Hanslmayr S, Matuschek J, Fellner MC (2014) Entrainment of prefrontal beta oscillations induces an endogenous echo and impairs memory formation. Curr Biol 24:904-909. CrossRef Medline

Herrmann CS, Rach S, Neuling T, Strüber D (2013) Transcranial alternating current stimulation: a review of the underlying mechanisms and modulation of cognitive processes. Front Hum Neurosci 7:279. CrossRef Medline

Ilmoniemi RJ, Kicić D (2010) Methodology for combined TMS and EEG. Brain Topogr 22:233-248. CrossRef Medline

Jaegle A, Ro T (2014) Direct control of visual perception with phase-specific modulation of posterior parietal cortex. J Cogn Neurosci 26:422-432. CrossRef Medline

Jensen O, Mazaheri A (2010) Shaping functional architecture by oscillatory alpha activity: gating by inhibition. Front Hum Neurosci 4:186. CrossRef Medline

Jensen O, Gips B, Bergmann TO, Bonnefond M (2014) Temporal coding organized by coupled alpha and gamma oscillations prioritize visual processing. Trends Neurosci 37:357-369. CrossRef Medline

Jung TP, Makeig S, Humphries C, Lee TW, McKeown MJ, Iragui V, Sejnowski TJ (2000) Removing electroencephalographic artifacts by blind source separation. Psychophysiology 37:163-178. CrossRef Medline

Klimesch W, Sauseng P, Gerloff C (2003) Enhancing cognitive performance with repetitive transcranial magnetic stimulation at human individual alpha frequency. Eur J Neurosci 17:1129-1133. CrossRef Medline

Klimesch W, Sauseng P, Hanslmayr S (2007) EEG alpha oscillations: the inhibition-timing hypothesis. Brain Res Rev 53:63-88. CrossRef Medline

Korhonen RJ, Hernandez-Pavon JC, Metsomaa J, Maki H, Ilmoniemi RJ, Sarvas J (2011) Removal of large muscle artifacts from transcranial magnetic stimulation-evoked EEG by independent component analysis. Med Biol Eng Comput 49:397-407. CrossRef Medline

Loftus GR, Masson MEJ (1994) Using confidence intervals in withinsubject designs. Psychonom Bull Rev 1:476-490. CrossRef Medline

Luck SJ (2005) An introduction to the event-related potential technique. Cambridge, MA: Massachusetts Institute of Technology.

Marshall TR, Bergmann TO, Jensen O (2015) Fronto-parietal structural connectivity mediates the top-down control of neuronal synchronization associated with selective attention. PLoS Biol 13:e1002272. CrossRef Medline

Massimini M, Ferrarelli F, Esser SK, Riedner BA, Huber R, Murphy M, Peterson MJ, Tononi G (2007) Triggering sleep slow waves by transcranial magnetic stimulation. Proc Natl Acad Sci U S A 104:8496-8501. CrossRef Medline

Mathewson KE, Gratton G, Fabiani M, Beck DM, Ro T (2009) To see or not to see: prestimulus alpha phase predicts visual awareness. J Neurosci 29: 2725-2732. CrossRef Medline

Mathewson KE, Lleras A, Beck DM, Fabiani M, Ro T, Gratton G (2011) Pulsed out of awareness: EEG alpha oscillations represent a pulsedinhibition of ongoing cortical processing. Front Psychol 2:99. CrossRef Medline

Mazaheri A, van Schouwenburg MR, Dimitrijevic A, Denys D, Cools R, Jensen O (2014) Region-specific modulations in oscillatory alpha activity serve to facilitate processing in the visual and auditory modalities. Neuroimage 87:356-362. CrossRef Medline

Moliadze V, Zhao Y, Eysel U, Funke K (2003) Effect of transcranial magnetic stimulation on single-unit activity in the cat primary visual cortex. J Physiol 553:665-679. CrossRef Medline

Oostendorp TF, van Oosterom A (1989) Source parameter estimation in inhomogeneous volume conductors of arbitrary shape. IEEE Trans Biomed Eng 36:382-391. Medline

Oostenveld R, Fries P, Maris E, Schoffelen JM (2011) FieldTrip: Open source software for advanced analysis of MEG, EEG, and invasive electrophysiological data. Comput Intell Neurosci 2011:156869. CrossRef Medline
Paus T, Sipila PK, Strafella AP (2001) Synchronization of neuronal activity in the human primary motor cortex by transcranial magnetic stimulation: an EEG study. J Neurophysiol 86:1983-1990. Medline

Perrin F, Pernier J, Bertrand O, Echallier JF (1989) Spherical splines for scalp potential and current density mapping. Electroencephalogr Clin Neurophysiol 72:184-187. CrossRef Medline

Perrin F, Pernier J, Bertrand O, Echallier JF (1990) Corrigenda: EEG 02274. Electroencephalogr Clin Neurophysiol 76:565-566. CrossRef

Premoli I, Castellanos N, Rivolta D, Belardinelli P, Bajo R, Zipser C, Espenhahn S, Heidegger T, Müller-Dahlhaus F, Ziemann U (2014) TMS-EEG signatures of GABAergic neurotransmission in the human cortex. J Neurosci 34:5603-5612. CrossRef Medline

Rajagovindan R, Ding M (2011) From prestimulus alpha oscillation to visual-evoked response: an inverted- $\mathrm{U}$ function and its attentional modulation. J Cogn Neurosci 23:1379-1394. CrossRef Medline

Romei V, Rihs T, Brodbeck V, Thut G (2008a) Resting electroencephalogram alpha-power over posterior sites indexes baseline visual cortex excitability. Neuroreport 19:203-208. CrossRef Medline

Romei V, Brodbeck V, Michel C, Amedi A, Pascual-Leone A, Thut G (2008b) Spontaneous fluctuations in posterior alpha-band EEG activity reflect variability in excitability of human visual areas. Cereb Cortex 18:20102018. CrossRef Medline

Romei V, Gross J, Thut G (2010) On the role of prestimulus alpha rhythms over occipito-parietal areas in visual input regulation: correlation or causation? J Neurosci 30:8692-8697. CrossRef Medline

Romei V, Driver J, Schyns PG, Thut G (2011) Rhythmic TMS over parietal cortex links distinct brain frequencies to global versus local visual processing. Curr Biol 21:334-337. CrossRef Medline

Romei V, Gross J, Thut G (2012) Sounds reset rhythms of visual cortex and corresponding human visual perception. Curr Biol 22:807-813. CrossRef Medline

Rosanova M, Casali A, Bellina V, Resta F, Mariotti M, Massimini M (2009) Natural frequencies of human corticothalamic circuits. J Neurosci 29: 7679-7685. CrossRef Medline

Ruzzoli M, Soto-Faraco S (2014) Alpha stimulation of the human parietal cortex attunes tactile perception to external space. Curr Biol 24:329-332. CrossRef Medline

Sauseng P, Klimesch W, Heise KF, Gruber WR, Holz E, Karim AA, Glennon M, Gerloff C, Birbaumer N, Hummel FC (2009) Brain oscillatory substrates of visual short-term memory capacity. Curr Biol 19:1846-1852. CrossRef Medline

Schroeder CE, Lakatos P (2009) Low-frequency neuronal oscillations as instruments of sensory selection. Trends Neurosci 32:9-18. CrossRef Medline

Thut G, Nietzel A, Brandt SA, Pascual-Leone A (2006) Alpha-band electroencephalographic activity over occipital cortex indexes visuospatial attention bias and predicts visual target detection. J Neurosci 26:9494-9502. CrossRef Medline

Thut G, Schyns PG, Gross J (2011a) Entrainment of perceptually relevant brain oscillations by non-invasive rhythmic stimulation of the human brain. Front Psychol 2:170. CrossRef Medline

Thut G, Veniero D, Romei V, Miniussi C, Schyns P, Gross J (2011b) Rhythmic TMS causes local entrainment of natural oscillatory signatures. Curr Biol 21:1176-1185. CrossRef Medline

Thut G, Miniussi C, Gross J (2012) The functional importance of rhythmic activity in the brain. Curr Biol 22:R658-R663. CrossRef Medline

VanRullen R, Koch C (2003) Is perception discrete or continuous? Trends Cogn Sci 7:207-213. CrossRef Medline

Varela F, Lachaux JP, Rodriguez E, Martinerie J (2001) The brainweb: phase synchronization and large-scale integration. Nat Rev Neurosci 2:229239. CrossRef Medline 